Pacific

Journal of

Mathematics

\title{
A CLASS OF PRIMARY REPRESENTATIONS ASSOCIATED WITH SYMMETRIC PAIRS AND RESTRICTED ROOT SYSTEMS
}

GANG HAN 


\title{
A CLASS OF PRIMARY REPRESENTATIONS ASSOCIATED WITH SYMMETRIC PAIRS AND RESTRICTED ROOT SYSTEMS
}

\author{
GANG HAN
}

\begin{abstract}
Let $v: \mathfrak{r} \rightarrow \mathfrak{s o}(\mathfrak{p})$ be a representation of a complex reductive Lie algebra $\mathfrak{r}$ on a complex vector space $\mathfrak{p}$. Assume that $v$ is the complexified differential of an orthogonal representation of a compact Lie group $R$. Then the exterior algebra $\Lambda \mathfrak{p}$ becomes an $\mathfrak{r}$-module by extending $v$. Let $\operatorname{Spin} v: \mathfrak{r} \rightarrow$ End $S$ be the composition of $v$ with the spin representation Spin : $\mathfrak{s o}(\mathfrak{p}) \rightarrow$ End $S$. We completely classify the representations $v$ for which the corresponding Spin $v$ representation is primary, give a description of the $\mathfrak{r}$-module structure of $\bigwedge \mathfrak{p}$, and present a decomposition of the Clifford algebra over $\mathfrak{p}$. It turns out that, if the Spin $v$ representation is primary, $v$ must be an isotropy representation of some symmetric pair. Our work generalizes Kostant's well-known results that dealt with the special case when $v$ is the adjoint representation of a semisimple Lie algebra. In the proof we introduce the "restricted" root system of a real semisimple Lie algebra, which is of independent interest.
\end{abstract}

\section{Introduction}

Let $R$ be a compact Lie group. Let $\mathfrak{p}$ be a finite-dimensional complex vector space with a nonsingular symmetric bilinear form $B_{\mathfrak{p}}$, and $C(\mathfrak{p})$ be the Clifford algebra over $\mathfrak{p}$ with respect to $B_{\mathfrak{p}}$. Assume that $v: R \rightarrow \mathrm{SO}(\mathfrak{p})$ is a $B_{\mathfrak{p}}$-orthogonal representation of $R$ on $\mathfrak{p}$. Let $\mathfrak{r}$ be the complexified Lie algebra of $R$. We use $v$ also to denote $v: \mathfrak{r} \rightarrow \mathfrak{s o}(\mathfrak{p})$, the complexified differential of the representation of $R$ on $\mathfrak{p}$. In particular, $\mathfrak{r}$ is reductive and $v$ is completely reducible. Let

$$
\beta: \mathfrak{r} \rightarrow \text { End } \wedge \mathfrak{p}
$$

be the derivation extension of $v$. Let

$$
\operatorname{Spin} v: \mathfrak{r} \rightarrow \text { End } S
$$

be the composition of $v$ with the spin representation $\operatorname{Spin}: \mathfrak{s o}(\mathfrak{p}) \rightarrow$ End $S$. When the representation Spin $v$ is primary of type $\pi_{\lambda}$, where $\pi_{\lambda}: \mathfrak{r} \rightarrow$ End $V_{\lambda}$ is the

Keywords: primary representation, spin representation, symmetric pair, restricted root system. 
irreducible representation of $\mathfrak{r}$ with highest weight $\lambda$, then, given the well-known relation between $\wedge \mathfrak{p}$ and $S \otimes S$, we have

$$
\bigwedge \mathfrak{p} \cong 2^{l} V_{\lambda} \otimes V_{\lambda}^{*}
$$

as $\mathfrak{r}$-modules, for some nonnegative integer $l$. One also has an algebra isomorphism

$$
C(\mathfrak{p}) \cong\left(\text { End } V_{\lambda}\right) \otimes J
$$

where $J=(\wedge \mathfrak{p})^{\mathfrak{r}}$, the space of $\mathfrak{r}$-invariants in $\bigwedge \mathfrak{p}$, is isomorphic to a matrix algebra if $l$ is even, and is isomorphic to a sum of two matrix algebras if $l$ is odd. See [Kostant 1997, Proposition 20].

In view of Equations (1-1) and (1-2), it is thus interesting to classify the representation $v$ such that $\operatorname{Spin} v$ is primary, which is the main result of this paper. Many people are interested in the special case where $v$ is the adjoint representation of a semisimple complex Lie algebra $\mathfrak{g}$ on itself. The study of an important graded submodule $C$ of $\wedge \mathfrak{g}$ can be found in [Kostant 2000; 1965]. The well-known result that the representation Spin $v$ is primary of type $\pi_{\rho}$, where $\rho$ is half the sum of the positive roots, was first given in [Kostant 1961]. Then the $\mathfrak{g}$-module structure of the exterior algebra $\bigwedge \mathfrak{g}$ is given by $\bigwedge \mathfrak{g} \cong 2^{l} V_{\rho} \otimes V_{\rho}$, where $l$ is the rank of $\mathfrak{g}$. The Clifford algebra $C(\mathfrak{g})$ over $\mathfrak{g}$ decomposes into the Clifford product

$$
C(\mathfrak{g}) \cong\left(\text { End } V_{\rho}\right) \otimes J
$$

where the space $J=(\bigwedge \mathfrak{g})^{\mathfrak{g}}$ has dimension $2^{l}$ and has a Clifford algebra structure over some subspace of itself.

In this paper we classify the representations $v$ such that the corresponding Spin $v$ is primary, and study the $\mathfrak{r}$-module structure of $\bigwedge \mathfrak{p}$ under the condition that Spin $v$ be primary. Our work generalizes the above results of Kostant's in the case of adjoint representations.

Recall that a Lie subalgebra $\mathfrak{k}$ of a Lie algebra $\mathfrak{g}$ is called a symmetric Lie subalgebra if there exists an involutory automorphism $\theta$ of $\mathfrak{g}$ such that $\mathfrak{k}$ is the set of $\theta$-invariants in $\mathfrak{g}$. In this case we call $(\mathfrak{k}, \mathfrak{g})$ a symmetric pair, and call the representation of $\mathfrak{k}$ on $\mathfrak{p} \cong \mathfrak{g} / \mathfrak{k}$ the isotropy representation of $(\mathfrak{k}, \mathfrak{g})$. It is surprising to us that if Spin $v$ is primary then $v$ must be the isotropy representation of some symmetric pair; see Proposition 2.4. Then, in order to classify the primary Spin $v$ representations, we need only consider isotropy representations of symmetric pairs.

Let $\mathfrak{g}_{0}$ be a noncompact real semisimple Lie algebra and $\mathfrak{g}_{0}=\mathfrak{k}_{0} \oplus \mathfrak{p}_{0}$ be a Cartan decomposition. Let $\mathfrak{g}=\mathfrak{k} \oplus \mathfrak{p}$ be its complexification and $\theta$ the corresponding involutory automorphism of $\mathfrak{g}$. Let $\mathfrak{h}_{0}=\mathfrak{t}_{0} \oplus \mathfrak{a}_{0}$ be a fundamental Cartan subalgebra of $\mathfrak{g}_{0}$, where $\mathfrak{t}_{0}$ is a Cartan subalgebra of $\mathfrak{k}_{0}$ and $\mathfrak{a}_{0}=\mathfrak{p}_{0}^{\mathfrak{t}_{0}}$, the centralizer of $\mathfrak{t}_{0}$ in $\mathfrak{p}_{0}$. Let $\mathfrak{h}=\mathfrak{t} \oplus \mathfrak{a}$ be its complexification. Let $l_{0}=\operatorname{dim} \mathfrak{a}$ and $r_{0}=\frac{1}{2} \operatorname{dim} \mathfrak{p} / \mathfrak{a}$, which 
will be shown to be an integer. For a complex vector space $V$, we will always use $\operatorname{dim} V$ to denote its complex dimension.

Let $\Delta(\mathfrak{g}, \mathfrak{h}) \subset \mathfrak{h}^{*}$ be the system of roots of $\mathfrak{g}$ with respect to $\mathfrak{h}$, and let $\mathfrak{h}_{\mathbb{R}}^{*}$ be the real span of the roots in $\Delta(\mathfrak{g}, \mathfrak{h})$. Usually "restricted roots" refers to the roots of $\mathfrak{g}_{0}$ with respect to a maximal abelian subspace of $\mathfrak{p}_{0}$, but in this paper, by abuse of language, we will call the roots of $\mathfrak{g}$ with respect to $\mathfrak{t}$ the restricted roots, and denote their set by $\Delta(\mathfrak{g}, \mathfrak{t})$. Let $\mathfrak{t}_{\mathbb{R}}^{*}$ be the real span of the roots in $\Delta(\mathfrak{g}, \mathfrak{t})$, which can be naturally identified with a subspace of $\mathfrak{h}_{\mathbb{R}}^{*}$. The restriction $B_{\mathfrak{g}} \mid \mathfrak{h}$ of the Killing form $B_{\mathfrak{g}}$ of $\mathfrak{g}$ is nonsingular and induces a symmetric nonsingular bilinear form $B_{\mathfrak{h}^{*}}$ on $\mathfrak{h}^{*}$. Because $B_{\mathfrak{h}^{*}}$ is positive definite on $\mathfrak{h}_{\mathbb{R}}^{*}$, it is also positive definite on $\mathfrak{t}_{\mathbb{R}}^{*}$. We will prove in Proposition 3.1 that the set $\Delta(\mathfrak{g}, \mathfrak{t})$ of restricted roots is a (maybe nonreduced) root system in $\mathfrak{t}_{\mathbb{R}}^{*}$.

In this paper we call $\Delta(\mathfrak{g}, \mathfrak{t})$ the restricted root system of $\mathfrak{g}_{0}$, which is independent of the $\mathfrak{t}_{0}$ chosen. Let $\Delta(\mathfrak{k}, \mathfrak{t})$ be the system of roots of $\mathfrak{k}$ with respect to $\mathfrak{t}$. Let $\Delta^{+}(\mathfrak{g}, \mathfrak{h})$ be a $\theta$-stable positive root system in $\Delta(\mathfrak{g}, \mathfrak{h})$. Let $\Gamma$ be the Dynkin diagram of $\Delta^{+}(\mathfrak{g}, \mathfrak{h})$. Then the involutory automorphism $\theta$ acts on $\Gamma$ naturally, and we get a pair $(\Gamma, \theta)$. The Dynkin diagram $\Gamma^{\prime}$ of $\Delta(\mathfrak{g}, \mathfrak{t})$ is completely determined by $(\Gamma, \theta)$. When $\mathfrak{g}$ is simple and $\theta$ is not the identity on $\Gamma$, we can get $\Gamma^{\prime}$ from $\Gamma$ easily; see Figure 1. These results suggest that the restricted root system $\Delta(\mathfrak{g}, \mathfrak{t})$ plays an important role in the structure of a real semisimple Lie algebra and deserves more attention.

There is an important result on the structure of the representation Spin $v$, where $v$ is the isotropy representation of a symmetric pair; see [Wallach 1988, Lemma 9.3.2]. We restate this result in terms of $\Delta(\mathfrak{k}, \mathfrak{t})$ and the restricted root system $\Delta(\mathfrak{g}, \mathfrak{t})$ in Lemma 4.3 , which reduces the problem of classification a lot. Then we first deal with the case when $\mathfrak{g}$ is simple. It is interesting that, for each connected Dynkin diagram with a nontrivial involutory automorphism, there is exactly one symmetric pair such that Spin $v$ is primary; see Proposition 4.7. Next we deal with the general case when $\mathfrak{g}$ is semisimple, and finish the classification completely in Theorem 4.13.

Let $\Delta^{+}(\mathfrak{k}, \mathfrak{t})$ be a positive root system of $\Delta(\mathfrak{k}, \mathfrak{t})$. Choose $\Delta^{+}(\mathfrak{g}, \mathfrak{t})$ to be a positive root system of $\Delta(\mathfrak{g}, \mathfrak{t})$ that contains $\Delta^{+}(\mathfrak{k}, \mathfrak{t})$. Define $\rho_{n}$ to be half the sum of roots in $\Delta^{+}(\mathfrak{g}, \mathfrak{t}) \backslash \Delta^{+}(\mathfrak{k}, \mathfrak{t})$. Combining Proposition 2.4 and Theorem 4.13, we get our main result, Theorem 4.14:

Theorem. Assume that $v: \mathfrak{r} \rightarrow \mathfrak{s o}(\mathfrak{p})$ is the complexified differential of a faithful $B_{\mathfrak{p}}$-orthogonal representation of a compact Lie group and that $\mathfrak{p}^{\mathfrak{r}}=0$, where $\mathfrak{p}^{\mathfrak{r}}$ are the $\mathfrak{r}$-invariants in $\mathfrak{p}$. Assume that $\operatorname{Spin} v$ is primary. Then $\mathfrak{g}=\mathfrak{r} \oplus \mathfrak{p}$ has a semisimple Lie algebra structure such that $\mathfrak{r}$ is a Lie subalgebra of $\mathfrak{g}$, that $(\mathfrak{r}, \mathfrak{g})$ is a reduced symmetric pair, and that $v$ is the isotropy representation of $(\mathfrak{r}, \mathfrak{g})$.

The symmetric pair $(\mathfrak{r}, \mathfrak{g})$ must be one of the following: 
(1) $(\mathfrak{s o}(2 n+1, \mathbb{C}), \mathfrak{s l}(2 n+1, \mathbb{C})), n \geq 1$; $(\mathfrak{s p}(n, \mathbb{C}), \mathfrak{s l}(2 n, \mathbb{C})), n \geq 2$ $(\mathfrak{s o}(2 n+1, \mathbb{C}), \mathfrak{s o}(2 n+2, \mathbb{C})), n \geq 3 ;$ $\left(F_{4}, E_{6}\right)$;

(2) $\mathfrak{g}=\mathfrak{g}_{1} \oplus \mathfrak{g}_{1}$ and $\mathfrak{r}=\left\{(X, X) \mid X \in \mathfrak{g}_{1}\right\}$, where $\mathfrak{g}_{1}$ is a complex simple Lie algebra;

(3) direct sums of $(\mathfrak{r}, \mathfrak{g})$ in (1) and (2).

Furthermore, when $(\mathfrak{r}, \mathfrak{g})$ is in this list, Spin $v$ is primary of type $\pi_{\rho_{n}}$.

Dmitri I. Panyushev [2001, Theorem 3.7] classified the primary Spin $v$ representations under the condition that $\mathfrak{r}$ is semisimple. Our assumption that $v$ is the complexified differential of an orthogonal representation of a compact Lie group is more general than his, although we did not get any new representation $v$ in the classification. Our approach is completely different from his and in our argument, based on results of Kostant, it is more direct to see that only isotropy representations of symmetric pairs may have primary Spin $v$ representations. It was also found in [Panyushev 2001] that when Spin $v$ is primary the space $(\wedge \mathfrak{p})^{\mathfrak{r}}$ of $\mathfrak{r}$-invariants in $\bigwedge \mathfrak{p}$ is still an exterior algebra.

Finally, as a corollary of [Kostant 1997, Proposition 20] — see Equations (1-1) and (1-2) - and recalling the definition of $l_{0}$ and $r_{0}$, we get a result on the $\mathfrak{r}$-module structure of $\bigwedge \mathfrak{p}$ and a decomposition of the Clifford algebra $C(\mathfrak{p})$, when Spin $v$ is primary:

Corollary 1.1. Let $(\mathfrak{r}, \mathfrak{g})$ be a symmetric pair in the list of the theorem above. The corresponding Spin $v$ representation is primary of type $\pi_{\rho_{n}}$. Let $J=(\wedge \mathfrak{p})^{\mathfrak{r}}$. Then $\operatorname{dim} V_{\rho_{n}}=2^{r_{0}}$ and $\operatorname{dim} J=2^{l_{0}}$. As a subalgebra of $C(\mathfrak{p}), J$ is isomorphic to a matrix algebra if $l_{0}$ is even, and is isomorphic to a sum of two matrix algebras if $l_{0}$ is odd. Furthermore,

$$
C(\mathfrak{p}) \cong\left(\text { End } V_{\rho_{n}}\right) \otimes J
$$

as algebras. Finally, as r-modules,

$$
\bigwedge \mathfrak{p} \cong 2^{l_{0}} V_{\rho_{n}} \otimes V_{\rho_{n}}
$$

\section{Preliminaries. A necessary condition for the representation Spin $v$ to be primary}

Let $\mathfrak{r}$ be a complex reductive Lie algebra and $B_{\mathfrak{r}}$ a nonsingular $\mathfrak{r}$-invariant symmetric bilinear form on $\mathfrak{r}$. Let $\mathfrak{p}$ be a finite-dimensional complex vector space with a nonsingular symmetric bilinear form $B_{\mathfrak{p}}$ on $\mathfrak{p}$. We always assume that

$$
v: \mathfrak{r} \rightarrow \mathfrak{s o}(\mathfrak{p})
$$


is a $B_{\mathfrak{p}}$-invariant representation of $\mathfrak{r}$ on $\mathfrak{p}$.

Next we recall some important relations arising from Chevalley's identification of the underlying vector spaces of the exterior algebra $\wedge \mathfrak{p}$ and the Clifford algebra $C(\mathfrak{p})$ over $\mathfrak{p}$ with respect to $B_{\mathfrak{p}}$. We think of $\Lambda \mathfrak{p}$ as having two multiplicative structures: $u w \in \wedge \mathfrak{p}$ denotes the Clifford product of $u$ and $w$, while $u \wedge w \in \wedge \mathfrak{p}$ denotes their exterior product. The natural extension of $B_{\mathfrak{p}}$ to $\wedge \mathfrak{p}$ is also denoted by $B_{\mathfrak{p}}$. For more details see [Kostant 1997, §2].

For any $u \in \bigwedge^{2} \mathfrak{p}$, let ad $u \in$ End $\bigwedge \mathfrak{p}$ be the operator defined by

$$
\operatorname{ad} u(w)=u w-w u
$$

for every $w \in \wedge \mathfrak{p}$. Then ad $u$ is not only a derivation of the Clifford algebra structure of $\wedge \mathfrak{p}$, but also a derivation of degree zero of the exterior algebra structure of $\wedge \mathfrak{p}$. In particular, $\bigwedge^{2} \mathfrak{p}$ is a Lie algebra under the Clifford product, and

$$
\operatorname{ad}: \bigwedge^{2} \mathfrak{p} \rightarrow \text { End } \wedge \mathfrak{p}, \quad u \mapsto \operatorname{ad} u
$$

is a Lie algebra representation. Furthermore,

$$
\tau: \bigwedge^{2} \mathfrak{p} \rightarrow \mathfrak{s o}(\mathfrak{p})
$$

is a Lie algebra isomorphism, where

$$
\tau(u)(x)=\operatorname{ad} u(x)
$$

for any $u \in \bigwedge^{2} \mathfrak{p}$ and $x \in \mathfrak{p}$. For proofs see [Kostant 1997, Proposition 7, Theorem 8].

It follows from (2-2) that there exists a unique Lie algebra homomorphism $\nu_{*}: \mathfrak{r} \rightarrow \bigwedge^{2} \mathfrak{p}$ such that

$$
\tau \circ \nu_{*}=v .
$$

Let

$$
\xi: \text { End } \mathfrak{p} \rightarrow \text { End } \wedge \mathfrak{p}
$$

be defined so that $\xi(z)$, for any $z \in$ End $\mathfrak{p}$, is the unique derivation in End $\wedge \mathfrak{p}$ that extends the action of $z$ to $\wedge \mathfrak{p}$. We also use $\xi$ to denote the above map restricted to $\mathfrak{s o}(\mathfrak{p})$. One knows that if $u \in \bigwedge^{2} \mathfrak{p}$ then $\operatorname{ad} u=\xi(\tau(u))$ (see [Kostant 1997, Theorem 8]), that is,

$$
\mathrm{ad}=\xi \circ \tau
$$

\section{Let}

$$
\beta: \mathfrak{r} \rightarrow \text { End } \bigwedge \mathfrak{p}
$$

be the composition of $v: \mathfrak{r} \rightarrow \mathfrak{s o}(\mathfrak{p})$ with $\xi: \mathfrak{s o}(\mathfrak{p}) \rightarrow$ End $\wedge \mathfrak{p}$. We will always refer to $\Lambda \mathfrak{p}$ as an $\mathfrak{r}$-module via $\beta$. The extended bilinear form $B_{\mathfrak{p}}$ is invariant under 
$\beta(x)$, for any $x \in \mathfrak{r}$. By Equations (2-3) and (2-4),

$$
\beta=\operatorname{adov} .
$$

Up to equivalence, the Clifford algebra $C(\mathfrak{p})$ has a unique faithful multiplicityfree module $S$. Let

$$
\varepsilon: C(\mathfrak{p}) \rightarrow \text { End } S
$$

be the corresponding homomorphism, and $S$ is referred to as the spin module for $C(\mathfrak{p})$. Let

$$
\text { Spin }: \mathfrak{s o}(\mathfrak{p}) \rightarrow \text { End } S
$$

be the composition of $\tau^{-1}: \mathfrak{s o}(\mathfrak{p}) \rightarrow \bigwedge^{2} \mathfrak{p}$ with $\varepsilon$. The composition of $\nu_{*}: \mathfrak{r} \rightarrow \bigwedge^{2} \mathfrak{p}$ with $\varepsilon$, or equivalently, the composition of $v: \mathfrak{r} \rightarrow \mathfrak{s o}(\mathfrak{p})$ with Spin, defines a representation

$$
\operatorname{Spin} v: \mathfrak{r} \rightarrow \text { End } S,
$$

called the spin of $\nu$. The maps above are organized into the commutative diagram

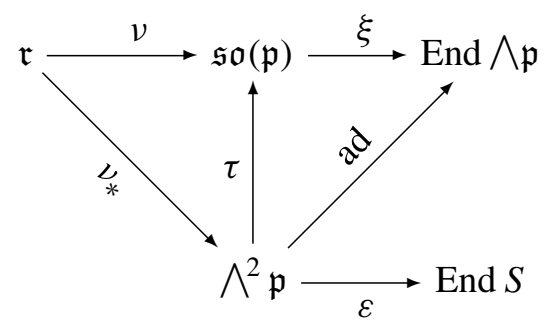

The underlying vector spaces of $\bigwedge \mathfrak{p}$ and $C(\mathfrak{p})$ are identified by Chevalley's map. When $\operatorname{dim} \mathfrak{p}$ is even,

$$
C(\mathfrak{p}) \cong \text { End } S
$$

as algebras, where $S$ is the spin module of $C(\mathfrak{p})$, which splits into two half-spin representations of $\bigwedge^{2} \mathfrak{p}(\cong \mathfrak{s o}(\mathfrak{p}))$ and is self-dual as a $\bigwedge^{2} \mathfrak{p}$-module. Under the adjoint representation ad, we have End $S \cong S \otimes S^{*}$ as $\bigwedge^{2}$ p-modules. Then, as $\bigwedge^{2} \mathfrak{p}$-modules, and hence as $\mathfrak{r}$-modules,

$$
\bigwedge \mathfrak{p} \cong S \otimes S
$$

When $\operatorname{dim} \mathfrak{p}$ is odd, the spin module $S$ of $C(\mathfrak{p})$ splits into two equivalent $\bigwedge^{2} \mathfrak{p}$ modules, $S_{1}$ and $S_{2}$. The space $S_{1}$ (or $S_{2}$ ) is called the spin representation of $\bigwedge^{2} \mathfrak{p}$ and is also self-dual. One has

$$
C(\mathfrak{p}) \cong \text { End } S_{1} \oplus \text { End } S_{2}
$$


as algebras. See, for example, $\S 3$ of [Kostant 1997] for details. Under the adjoint action ad, we have End $S \cong S_{1} \otimes S_{1}^{*} \oplus S_{2} \otimes S_{2}^{*} \cong 2 S_{1} \otimes S_{1}$ as $\wedge^{2} \mathfrak{p}$-modules. Then, as $\bigwedge^{2} \mathfrak{p}$-modules, and hence as $\mathfrak{r}$-modules, $\bigwedge \mathfrak{p} \cong 2 S_{1} \otimes S_{1}$, or

$$
S \otimes S \cong 2 \wedge \mathfrak{p}
$$

Recall that a completely reducible representation $\pi: \mathfrak{r} \rightarrow$ End $V$ is primary if there exists a representation $\pi_{\lambda}: \mathfrak{r} \rightarrow$ End $V_{\lambda}$, the irreducible representation of $\mathfrak{r}$ with highest weight $\lambda$, such that every irreducible component of $\pi$ is equivalent to $\pi_{\lambda}$. More specifically, in such a case we say that $\pi$ is primary of type $\pi_{\lambda}$.

The relations (2-9) and (2-11) imply that, if $S$ is primary of type $\pi_{\lambda}$, then

$$
\bigwedge \mathfrak{p} \cong 2^{l} V_{\lambda} \otimes V_{\lambda}
$$

as $\mathfrak{r}$-modules, for some nonnegative integer $l$.

Let $\nu_{*}: U(\mathfrak{r}) \rightarrow C^{0}(\mathfrak{p})$ be the algebra homomorphism extending $v_{*}: \mathfrak{r} \rightarrow \bigwedge^{2} \mathfrak{p}$, where $U(\mathfrak{r})$ is the universal enveloping algebra of $\mathfrak{r}$. Let $E$ be the image of $U(\mathfrak{r})$ and let $J=(\bigwedge \mathfrak{p})^{\mathfrak{r}}$. Then $J$ equals the centralizer of $E$ in $C(\mathfrak{p})$. If $S$ is primary of type $\pi_{\lambda}$, one has

$$
C(\mathfrak{p}) \cong E \otimes J
$$

as algebras, and $E \cong$ End $V_{\lambda}$. See [Kostant 1997, Proposition 20].

Let $\mathfrak{g}$ be a complex Lie algebra and let $\theta$ be an involutory automorphism of $\mathfrak{g}$. Then $(\mathfrak{g}, \theta)$ is called an involutory complex Lie algebra, and $\theta$ is referred to as the corresponding Cartan involution. Let $\mathfrak{g}=\mathfrak{k} \oplus \mathfrak{p}$ be the Cartan decomposition of $\mathfrak{g}$ into $(+1)$ - and $(-1)$-eigenspaces of $\theta$. A Lie subalgebra $\mathfrak{k}$ is called a symmetric Lie subalgebra of $\mathfrak{g}$ if there exists an involutory automorphism $\theta$ of $\mathfrak{g}$ such that $\mathfrak{k}$ is the set of $\theta$-invariants in $\mathfrak{g}$. In this case $(\mathfrak{k}, \mathfrak{g})$ is called a symmetric pair.

Two involutory Lie algebra $\left(\mathfrak{g}_{1}, \theta_{1}\right)$ and $\left(\mathfrak{g}_{2}, \theta_{2}\right)$ are isomorphic if there exists an isomorphism $\psi: \mathfrak{g}_{1} \rightarrow \mathfrak{g}_{2}$ satisfying $\psi \theta_{1}=\theta_{2} \psi$. In fact, since $\mathfrak{k}$ and $\theta$ uniquely determine each other, we will often refer to either as corresponding to the other. We say that two symmetric pairs are isomorphic if their corresponding involutory Lie algebras are isomorphic. Let $\mathrm{ad}_{\mathfrak{g}}$ denote the adjoint representation of $\mathfrak{g}$ on itself. Since $[\mathfrak{k}, \mathfrak{p}] \subseteq \mathfrak{p}$, we can define

$$
\mathfrak{k} \rightarrow \text { End } \mathfrak{p}, \quad x \mapsto\left(\operatorname{ad}_{\mathfrak{g}} x\right) \mid \mathfrak{p}
$$

for any $x \in \mathfrak{k}$; we call it the isotropy representation of the symmetric pair $(\mathfrak{k}, \mathfrak{g})$. It is easy to see that, for isomorphic symmetric pairs, the corresponding symmetric Lie subalgebras $\mathfrak{k}$ are isomorphic and the corresponding isotropy representations are equivalent. The involutory complex Lie algebra $(\mathfrak{g}, \theta)$ and the symmetric pair $(\mathfrak{k}, \mathfrak{g})$ are said to be reduced if $\mathfrak{k}$ contains no nonzero ideal of $\mathfrak{g}$. 
Recall that $v: \mathfrak{r} \rightarrow \mathfrak{s o}(\mathfrak{p})$ is a $B_{\mathfrak{p}}$-invariant representation of $\mathfrak{r}$ on $\mathfrak{p}$, where $\mathfrak{r}$ is a complex reductive Lie algebra with a nonsingular ad $\mathfrak{r}$-invariant symmetric bilinear form $B_{\mathfrak{r}}$ and $\mathfrak{p}$ is a complex vector space with a nonsingular symmetric bilinear form $B_{\mathfrak{p}}$.

Let $\mathfrak{g}=\mathfrak{r} \oplus \mathfrak{p}$ and let $B_{\mathfrak{g}}$ be the nonsingular symmetric bilinear form on $\mathfrak{g}$ defined so that $B_{\mathfrak{g}}\left|\mathfrak{r}=B_{\mathfrak{r}}, B_{\mathfrak{g}}\right| \mathfrak{p}=B_{\mathfrak{p}}$, and $\mathfrak{p}$ is $B_{\mathfrak{g}}$-orthogonal to $\mathfrak{r}$. As in [Kostant 1999, $\S 1.1]$, we say that the representation $\left(\nu, B_{\mathfrak{g}}\right)$ is of Lie type if there exists a Lie algebra structure [, ] on $\mathfrak{g}$ such that $\mathfrak{r}$ is a Lie subalgebra of $\mathfrak{g}$ and $[x, y]=v(x) y$ for $x \in \mathfrak{r}, y \in \mathfrak{p}$, and if moreover $B_{\mathfrak{g}}$ is $\mathfrak{g}$-invariant. Let $\operatorname{Cas}_{\mathfrak{r}}$ denote the Casimir element of $\mathfrak{r}$ with respect to $B_{\mathfrak{r}}$.

Kostant proved an important relation between the representations Spin $v$ and $v$ :

Theorem 2.1 [Kostant 1999]. The following conditions are equivalent:

(1) $(\operatorname{Spin} v)\left(\mathrm{Cas}_{\mathfrak{r}}\right)$ is a scalar multiple of the identity operator on $S$;

(2) $\left(v, B_{\mathfrak{g}}\right)$ is of Lie type and $(\mathfrak{r}, \mathfrak{g})$ is a symmetric pair, where $\mathfrak{p}$ is the (-1)eigenspace for a corresponding Cartan involution.

Remark 2.2. There are four equivalent conditions listed in [Kostant 1999, Theorem 1.59], but for our purpose we only list two of them here. When any of the conditions of the theorem are satisfied, the Lie algebra structure of $\mathfrak{g}=\mathfrak{r} \oplus \mathfrak{p}$ is determined in $\S 1$ of [Kostant 1999].

Theorem 2.3 [Kostant 1999, Theorem 1.61]. Assume that $v$ is the complexified differential of a faithful $B_{\mathfrak{p}}$-orthogonal representation of a compact Lie group and that $\mathfrak{p}^{\mathfrak{r}}=0$. Assume that any of the conditions of Theorem 2.1 is satisfied. Then $\mathfrak{g}$ is semisimple, and $\mathfrak{p}$ is the orthogonal complement of $\mathfrak{r}$ in $\mathfrak{g}$ with respect to the Killing form.

Our desired result follows as a corollary:

Proposition 2.4. Assume that $v: \mathfrak{r} \rightarrow \mathfrak{s o}(\mathfrak{p})$ is the complexified differential of a faithful $B_{\mathfrak{p}}$-orthogonal representation of a compact Lie group and that $\mathfrak{p}^{\mathfrak{r}}=0$. If Spin $v$ is primary, then $\left(v, B_{\mathfrak{g}}\right)$ is of Lie type, $(\mathfrak{r}, \mathfrak{g})$ is a reduced symmetric pair with $\mathfrak{g}$ semisimple, and $v$ is the isotropy representation of $(\mathfrak{r}, \mathfrak{g})$.

Proof. If the representation Spin $v$ is primary, say, of type $\pi_{\lambda}$, then $(\operatorname{Spin} v)\left(\operatorname{Cas}_{\mathfrak{r}}\right)$ is a scalar multiple of the identity operator on $S$, because $\operatorname{Cas}_{\mathfrak{r}}$ is in the center of $U(\mathfrak{r})$. Then, by Theorem $2.1,\left(\nu, B_{\mathfrak{g}}\right)$ is of Lie type and $(\mathfrak{r}, \mathfrak{g})$ is a symmetric pair where $\mathfrak{p}$ is the $(-1)$-eigenspace for a corresponding Cartan involution. So $v$ is the isotropy representation of $(\mathfrak{r}, \mathfrak{g})$. The symmetric pair $(\mathfrak{r}, \mathfrak{g})$ is reduced because $v$ is faithful. Since all the assumptions of Theorem 2.3 are satisfied, $\mathfrak{g}$ is semisimple.

Hence, in order to classify all the primary Spin $v$ representations, we need only consider the isotropy representations of symmetric pairs $(\mathfrak{k}, \mathfrak{g})$ with $\mathfrak{g}$ semisimple. 


\section{Restricted root system of a real semisimple Lie algebra}

Let $(\mathfrak{g}, \theta)$ be an involutory complex semisimple Lie algebra. Let $\mathfrak{g}=\mathfrak{k} \oplus \mathfrak{p}$ be the corresponding Cartan decomposition. Let $B_{\mathfrak{g}}$ be an $\mathfrak{g}$-invariant nonsingular symmetric bilinear form on $\mathfrak{g}$. Assume that the isotropy representation $v: \mathfrak{k} \rightarrow$ $\mathfrak{s o}(\mathfrak{p})$ is faithful, which is equivalent to $(\mathfrak{k}, \mathfrak{g})$ being reduced. The $\mathfrak{k}$-module $\mathfrak{p}$ is completely reducible and there is no nonzero trivial submodule of $\mathfrak{p}$; hence the space $\mathfrak{p}^{\mathfrak{k}}$ of $\mathfrak{k}$ invariants in $\mathfrak{p}$ is always 0 . Now our objective is to classify all the reduced symmetric pairs $(\mathfrak{k}, \mathfrak{g})$ such that the corresponding $\operatorname{Spin} v$ representation is primary. Considering that $v$ is completely reducible, it is not hard to see that the $\mathfrak{k}$-module structure of $S$ does not depend on the nonsingular $\mathfrak{g}$-invariant symmetric bilinear form $B_{\mathfrak{g}}$. So from now on we will just assume $B_{\mathfrak{g}}$ to be the Killing form on $\mathfrak{g}$.

For a noncompact real semisimple Lie algebra $\mathfrak{g}_{0}$, let $\mathfrak{g}_{0}=\mathfrak{k}_{0} \oplus \mathfrak{p}_{0}$ be a Cartan decomposition of $\mathfrak{g}_{0}$. Let $\mathfrak{g}=\mathfrak{k} \oplus \mathfrak{p}$ be its complexification and $\theta$ the corresponding involution of $\mathfrak{g}$. Then $(\mathfrak{g}, \theta)$ is an involutory complex semisimple Lie algebra. Conversely, for a complex semisimple involutory Lie algebra $(\mathfrak{g}, \theta)$, there exists a real form $\mathfrak{g}_{0}$ of $\mathfrak{g}$ such that $\theta \mid \mathfrak{g}_{0}$ is a Cartan involution of $\mathfrak{g}_{0}$. Up to isomorphism, $\mathfrak{g}_{0}$ and $(\mathfrak{g}, \theta)$ uniquely determine each other. Let $\mathfrak{h}_{0}=\mathfrak{t}_{0} \oplus \mathfrak{a}_{0}$ be a maximally compact Cartan subalgebra of $\mathfrak{g}_{0}$, that is, $\mathfrak{t}_{0}$ is a Cartan subalgebra of $\mathfrak{k}_{0}$ and $\mathfrak{a}_{0}=\mathfrak{p}_{0}^{\mathfrak{t}_{0}}$. Let $\mathfrak{h}=\mathfrak{t} \oplus \mathfrak{a}$ be its complexification, which is also a Cartan subalgebra of $\mathfrak{g}$. We call such an $\mathfrak{h}$ a maximally compact Cartan subalgebra of $(\mathfrak{g}, \theta)$. Let $l_{0}=\operatorname{dim} \mathfrak{a}$. Let

$$
\mathfrak{h}_{\mathbb{R}}=i \mathfrak{t}_{0} \oplus \mathfrak{a}_{0},
$$

which is a real form of $\mathfrak{h}$.

Let $\Delta(\mathfrak{g}, \mathfrak{h}) \subset \mathfrak{h}^{*}$ be the set of roots of $\mathfrak{g}$ with respect to $\mathfrak{h}$. Obviously, we have $\theta(\Delta(\mathfrak{g}, \mathfrak{h}))=\Delta(\mathfrak{g}, \mathfrak{h})$. Let $\mathfrak{h}_{\mathbb{R}}^{*}$ be the real span of the roots in $\Delta(\mathfrak{g}, \mathfrak{h})$, so that $\mathfrak{h}_{\mathbb{R}}^{*}$ is a real form of $\mathfrak{h}^{*}$ and can be taken as the real dual space to $\mathfrak{h}_{\mathbb{R}}$, because the roots in $\Delta(\mathfrak{g}, \mathfrak{h})$ take real values on $\mathfrak{h}_{\mathbb{R}}$. Let $\theta$ act on $\mathfrak{h}^{*}$ by $(\theta \sigma)(H)=\sigma(\theta H)$, where $\sigma \in \mathfrak{h}^{*}, H \in \mathfrak{h}$. Clearly $\mathfrak{h}_{\mathbb{R}}^{*}$ is $\theta$-stable. Let

$$
\mathfrak{h}_{\mathbb{R}}^{*}=\mathfrak{t}_{\mathbb{R}}^{*} \oplus \mathfrak{a}_{\mathbb{R}}^{*}
$$

be the decomposition of $\mathfrak{h}_{\mathbb{R}}^{*}$ into (+1)- and (-1)-eigenspaces of $\theta$. Let

$$
p: \mathfrak{h}_{\mathbb{R}}^{*} \rightarrow \mathfrak{t}_{\mathbb{R}}^{*}
$$

be the projection of $\mathfrak{h}_{\mathbb{R}}^{*}$ onto $\mathfrak{t}_{\mathbb{R}}^{*}$.

Set $\Delta(\mathfrak{g}, \mathfrak{t})=\{p(\alpha) \mid \alpha \in \Delta(\mathfrak{g}, \mathfrak{h})\}$. Then $\mathfrak{t}_{\mathbb{R}}^{*}$ is the real span of the roots in $\Delta(\mathfrak{g}, \mathfrak{t})$. The restriction $B_{\mathfrak{g}} \mid \mathfrak{h}$ is nonsingular and induces a symmetric nonsingular bilinear form $B_{\mathfrak{h}^{*}}$ on $\mathfrak{h}^{*}$. One knows that $B_{\mathfrak{h}^{*}}$ is positive definite on $\mathfrak{h}_{\mathbb{R}}^{*}$; hence 
also on $\mathfrak{t}_{\mathbb{R}}^{*}$. Then (3-1) is clearly an orthogonal direct-sum decomposition, and $p(\alpha)=(\alpha+\theta(\alpha)) / 2$ for $\alpha \in \mathfrak{h}_{\mathbb{R}}^{*}$. Let $(\gamma, \delta)$ denote the value of $B_{\mathfrak{h}^{*}}$ on $\gamma, \delta \in \mathfrak{h}_{\mathbb{R}}^{*}$.

For any $\alpha, \gamma \in \Delta(\mathfrak{g}, \mathfrak{h})$ or $\alpha, \gamma \in \Delta(\mathfrak{g}, \mathfrak{t})$, define

$$
\langle\alpha, \gamma\rangle=\frac{2(\alpha, \gamma)}{(\gamma, \gamma)}
$$

It is well-known that $\Delta(\mathfrak{g}, \mathfrak{h})$ is a root system in $\mathfrak{h}_{\mathbb{R}}^{*}$. Moreover:

Proposition 3.1. The set $\Delta(\mathfrak{g}, \mathfrak{t})$ is a root system (maybe nonreduced) in $\mathfrak{t}_{\mathbb{R}}^{*}$.

Proof. For any $\alpha \in \Delta(\mathfrak{g}, \mathfrak{h})$, we use $\alpha^{\prime}$ to denote $p(\alpha) \in \Delta(\mathfrak{g}, \mathfrak{t})$. Then $\left(\alpha^{\prime}, \gamma^{\prime}\right)=$ $\left(\alpha, \gamma^{\prime}\right)$ as $\left(\alpha^{\prime}-\alpha, \gamma^{\prime}\right)=0$. For any $\alpha, \gamma \in \Delta(\mathfrak{g}, \mathfrak{h})$ (respectively, any $\left.\alpha, \gamma \in \Delta(\mathfrak{g}, \mathfrak{t})\right)$, define $s_{\gamma} \alpha=\alpha-\langle\alpha, \gamma\rangle \gamma$, the reflection of $\alpha$ along $\gamma$. Then we need only show that, for any $\alpha^{\prime}, \gamma^{\prime} \in \Delta(\mathfrak{g}, \mathfrak{t})$, we have $\left\langle\alpha^{\prime}, \gamma^{\prime}\right\rangle \in \mathbb{Z}$ and $s_{\gamma^{\prime}} \alpha^{\prime} \in \Delta(\mathfrak{g}, \mathfrak{t})$. We distinguish two cases.

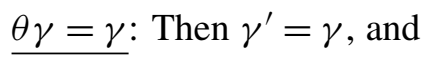

$$
\left\langle\alpha^{\prime}, \gamma^{\prime}\right\rangle=\frac{2\left(\alpha^{\prime}, \gamma\right)}{(\gamma, \gamma)}=\frac{2(\alpha, \gamma)}{(\gamma, \gamma)}=\langle\alpha, \gamma\rangle \in \mathbb{Z}
$$

Thus, $s_{\gamma^{\prime}} \alpha^{\prime}=\alpha^{\prime}-\left\langle\alpha^{\prime}, \gamma^{\prime}\right\rangle \gamma^{\prime}=\alpha^{\prime}-\langle\alpha, \gamma\rangle \gamma^{\prime}=\left(s_{\gamma} \alpha\right)^{\prime}$.

$\theta \gamma \neq \gamma$ : Since $\mathfrak{h}$ is a maximally compact Cartan subalgebra of $(\mathfrak{g}, \theta)$, there is no real root (that is, vanishing on $\mathfrak{t}$ ) in $\Delta(\mathfrak{g}, \mathfrak{h})$. So $(\gamma, \theta \gamma) \leq 0$, as otherwise $\gamma-\theta \gamma$ will be a real root.

If $(\gamma, \theta \gamma)<0$, then $\gamma+\theta \gamma=2 \gamma^{\prime}$ is a root in $\Delta(\mathfrak{g}, \mathfrak{h})$ and also in $\Delta(\mathfrak{g}, \mathfrak{t})$. Then $s_{\gamma^{\prime}} \alpha^{\prime}=s_{\gamma+\theta \gamma} \alpha^{\prime}=\left(s_{\gamma+\theta \gamma} \alpha\right)^{\prime} \in \Delta(\mathfrak{g}, \mathfrak{t})$. But $\left(s_{\gamma+\theta \gamma} \alpha\right)^{\prime}=\alpha^{\prime}-2\langle\alpha, \gamma+\theta \gamma\rangle \gamma^{\prime}$, and so $\left\langle\alpha^{\prime}, \gamma^{\prime}\right\rangle=2\langle\alpha, \gamma+\theta \gamma\rangle \in \mathbb{Z}$.

If $(\gamma, \theta \gamma)=0$, then $\left(\gamma^{\prime}, \gamma^{\prime}\right)=(\gamma, \gamma) / 2$. By computation,

$$
s_{\gamma^{\prime}} \alpha^{\prime}=\alpha^{\prime}-\frac{2(\alpha, \gamma+\theta \gamma)}{(\gamma, \gamma)} \gamma^{\prime}=\left(s_{\gamma} s_{\theta \gamma} \alpha\right)^{\prime} \text {. }
$$

In this case

$$
\left\langle\alpha^{\prime}, \gamma^{\prime}\right\rangle=\frac{2(\alpha, \gamma+\theta \gamma)}{(\gamma, \gamma)}=\frac{2(\alpha+\theta \alpha, \gamma)}{(\gamma, \gamma)}=\langle\alpha, \gamma\rangle+\langle\theta \alpha, \gamma\rangle
$$

is also an integer.

Remark 3.2. In this paper we call $\Delta(\mathfrak{g}, \mathfrak{t})$ the restricted root system of $\mathfrak{g}_{0}$, which is clearly independent of the Cartan subalgebra $\mathfrak{t}_{0}$ of $\mathfrak{k}_{0}$ chosen. For a $\theta$-stable positive root system $\Delta^{+}(\mathfrak{g}, \mathfrak{h})$ of $\Delta(\mathfrak{g}, \mathfrak{h})$, it is obvious that $p\left(\Delta^{+}(\mathfrak{g}, \mathfrak{h})\right)$ is also a positive root system in $\Delta(\mathfrak{g}, \mathfrak{t})$. Furthermore, for the set of simple roots $\Pi$ of $\Delta^{+}(\mathfrak{g}, \mathfrak{h})$, $p(\Pi)$ is also the set of simple roots in $p\left(\Delta^{+}(\mathfrak{g}, \mathfrak{h})\right)$. Conversely, if $\Delta^{+}(\mathfrak{g}, \mathfrak{t})$ is a positive root system in $\Delta(\mathfrak{g}, \mathfrak{t})$, then $p^{-1}\left(\Delta^{+}(\mathfrak{g}, \mathfrak{t})\right)$ is a $\theta$-stable positive root system of $\Delta(\mathfrak{g}, \mathfrak{h})$. Consequently, the $\theta$-stable positive root systems of $\Delta(\mathfrak{g}, \mathfrak{h})$ 
and the positive root systems of $\Delta(\mathfrak{g}, \mathfrak{t})$ are in one-to-one correspondence under $p$. Let $W(\Delta(\mathfrak{g}, \mathfrak{h}))$ be the Weyl group of $\Delta(\mathfrak{g}, \mathfrak{h})$. The Weyl group of $\Delta(\mathfrak{g}, \mathfrak{t})$ is in fact isomorphic to the subgroup of $W(\Delta(\mathfrak{g}, \mathfrak{h}))$ consisting of those elements of $W(\Delta(\mathfrak{g}, \mathfrak{h}))$ commuting with $\theta$.

Let $\mathfrak{g}_{\alpha}$ be the one-dimensional root space corresponding to $\alpha$. Let

$$
\begin{aligned}
& \Delta_{1}=\left\{\alpha \in \Delta(\mathfrak{g}, \mathfrak{h}) \mid \mathfrak{g}_{\alpha} \subset \mathfrak{k}\right\}, \\
& \Delta_{2}=\left\{\alpha \in \Delta(\mathfrak{g}, \mathfrak{h}) \mid \mathfrak{g}_{\alpha} \subset \mathfrak{p}\right\}, \\
& \Delta_{3}=\Delta(\mathfrak{g}, \mathfrak{h}) \backslash\left(\Delta_{1} \cup \Delta_{2}\right) .
\end{aligned}
$$

The roots in $\Delta_{1}$ and $\Delta_{2}$ are called imaginary. We call an imaginary root compact if $\alpha \in \Delta_{1}$ and noncompact if $\alpha \in \Delta_{2}$. The roots in $\Delta_{3}$ are called complex. Then $\Delta(\mathfrak{g}, \mathfrak{h})=\Delta_{1} \sqcup \Delta_{2} \sqcup \Delta_{3}$. We choose a positive root system $\Delta^{+}(\mathfrak{g}, \mathfrak{h})$ such that $\theta\left(\Delta^{+}(\mathfrak{g}, \mathfrak{h})\right)=\Delta^{+}(\mathfrak{g}, \mathfrak{h})$. Let $\Pi$ be the set of simple roots in $\Delta^{+}(\mathfrak{g}, \mathfrak{h})$. Then $\theta$ permutes the simple roots in $\Pi$ and induces an involutory automorphism of the Dynkin diagram $\Gamma$ of $\Pi$. For the complex semisimple involutory Lie algebra $(\mathfrak{g}, \theta)$, by abuse of language, we call $(\Gamma, \theta)$ its Dynkin diagram. We also call $(\Gamma, \theta)$ the Dynkin diagram of the corresponding $\mathfrak{g}_{0}$. Obviously, $\theta$ fixes the imaginary simple roots in $\Gamma$ and permutes in 2-cycles the complex simple roots in $\Gamma$.

Lemma 3.3. The Dynkin diagram $(\Gamma, \theta)$ of $(\mathfrak{g}, \theta)$ is independent of the $\theta$-stable positive root system chosen.

Proof. Assume that $\Delta_{1}^{+}$and $\Delta_{2}^{+}$are two $\theta$-stable positive root systems of $\Delta(\mathfrak{g}, \mathfrak{h})$. For $i=1$ or 2 , let $\Pi_{i}$ be the set of simple roots in $\Delta_{i}^{+}$and $\Gamma_{i}$ the Dynkin diagram of $\Pi_{i}$. Then there exists $\phi \in W(\Delta(\mathfrak{g}, \mathfrak{h}))$, the Weyl group of $\Delta(\mathfrak{g}, \mathfrak{h})$, such that $\phi\left(\Delta_{1}^{+}\right)=\Delta_{2}^{+}$. One also has $\phi\left(\Pi_{1}\right)=\Pi_{2}$. Then $\phi \theta\left(\Delta_{1}^{+}\right)=\theta \phi\left(\Delta_{1}^{+}\right)$, so $\phi^{-1} \theta^{-1} \phi \theta\left(\Delta_{1}^{+}\right)=\Delta_{1}^{+}$. Since $W(\Delta(\mathfrak{g}, \mathfrak{h}))$ is a normal subgroup of the automorphism group of $\Delta(\mathfrak{g}, \mathfrak{h})$, one has $\phi^{-1} \theta^{-1} \phi \theta \in W(\Delta(\mathfrak{g}, \mathfrak{h}))$. Because $W(\Delta(\mathfrak{g}, \mathfrak{h}))$ acts simply transitively on the set of positive root systems, $\phi^{-1} \theta^{-1} \phi \theta=1$. So $\phi \theta=\theta \phi$. Hence, under $\phi$ the actions of $\theta$ on $\Delta_{1}^{+}$and $\Delta_{2}^{+}$are equivalent. Therefore $\left(\Gamma_{1}, \theta\right)$ and $\left(\Gamma_{2}, \theta\right)$ are the same.

Note that $(\Gamma, \theta)$ gives less information for $\mathfrak{g}_{0}$ than the Vogan diagram of $\mathfrak{g}_{0}$, and nonisomorphic $\mathfrak{g}_{0}$ 's may have the same $(\Gamma, \theta)$.

For any $\alpha \in \Delta(\mathfrak{g}, \mathfrak{t})$, also let

$$
\mathfrak{g}_{\alpha}=\{X \in \mathfrak{g} \mid[H, X]=\alpha(H) X \text { for all } H \in \mathfrak{t}\}
$$

be the root space corresponding to $\alpha$. The multiplicity of $\alpha \in \Delta(\mathfrak{g}, \mathfrak{t})$ is defined to be the dimension of $\mathfrak{g}_{\alpha}$. Let $\Delta(\mathfrak{k}, \mathfrak{t}) \subset \mathfrak{t}^{*}$ be the set of roots of $\mathfrak{k}$ with respect to $\mathfrak{t}$. Define

$$
\Delta(\mathfrak{p}, \mathfrak{t})=\left\{\alpha \in \Delta(\mathfrak{g}, \mathfrak{t}) \mid \mathfrak{g}_{\alpha} \cap \mathfrak{p} \neq 0\right\}
$$


Then $\Delta(\mathfrak{g}, \mathfrak{t})=\Delta(\mathfrak{k}, \mathfrak{t}) \cup \Delta(\mathfrak{p}, \mathfrak{t})$. For any $\alpha \in \Delta(\mathfrak{p}, \mathfrak{t})$, let $\mathfrak{p}_{\alpha}=\mathfrak{g}_{\alpha} \cap \mathfrak{p}$. Then

$$
\mathfrak{p}=\mathfrak{a} \oplus \sum_{\alpha \in \Delta(\mathfrak{p}, \mathfrak{t})} \mathfrak{p}_{\alpha}
$$

$\Delta(\mathfrak{p}, \mathfrak{t})$ is just the set of nonzero weights of $\mathfrak{p}$ with respect to the Cartan subalgebra $\mathfrak{t}$ of $\mathfrak{k}$, because $\mathfrak{p}$ is the space of the isotropy representation of $\mathfrak{k}$.

Lemma 3.4. $\mathfrak{p}_{\alpha}$ is one-dimensional, for any $\alpha \in \Delta(\mathfrak{p}, \mathfrak{t})$.

Proof. If $\alpha \in \Delta_{2}$, then $\mathfrak{p}_{\alpha}=\mathfrak{g}_{\alpha}$ is one-dimensional. Otherwise, assume that $\alpha=$ $p(\gamma)$ for some $\gamma \in \Delta_{3}$. Because there are no real roots in $\Delta(\mathfrak{g}, \mathfrak{h})$, we see that $p(\gamma) \neq p(\alpha)$, for any $\alpha, \gamma \in \Delta(\mathfrak{g}, \mathfrak{h})$ such that $\gamma \neq \alpha$ and $\gamma \neq \theta \alpha$. Then

$$
\mathfrak{g}_{\alpha}=\sum_{\substack{\beta \in \Delta(\mathfrak{g}, \mathfrak{h}) \\ p(\beta)=\alpha}} \mathfrak{g}_{\beta}=\mathfrak{g}_{\gamma} \oplus \mathfrak{g}_{\theta \gamma} .
$$

Let $X_{\gamma}$ be a root vector of $\gamma$. Then $\theta X_{\gamma}$ is a root vector of $\theta \gamma$. So both $\mathfrak{k}_{\alpha}=$ $\mathfrak{g}_{\alpha} \cap \mathfrak{k}=\mathbb{C}\left(X_{\gamma}+\theta X_{\gamma}\right)$ and $\mathfrak{p}_{\alpha}=\mathbb{C}\left(X_{\gamma}-\theta X_{\gamma}\right)$ are one-dimensional.

The proof of the lemma also implies that

$$
\begin{aligned}
& \Delta(\mathfrak{k}, \mathfrak{t})=\Delta_{1} \sqcup p\left(\Delta_{3}\right), \\
& \Delta(\mathfrak{p}, \mathfrak{t})=\Delta_{2} \sqcup p\left(\Delta_{3}\right), \\
& \Delta(\mathfrak{k}, \mathfrak{t}) \cap \Delta(\mathfrak{p}, \mathfrak{t})=p\left(\Delta_{3}\right) .
\end{aligned}
$$

The multiplicity of $\alpha \in \Delta(\mathfrak{g}, \mathfrak{t})$ (that is, the dimension of $\mathfrak{g}_{\alpha}$ ) is 1 if $\alpha \in \Delta_{1} \sqcup \Delta_{2}$, and is 2 if $\alpha \in p\left(\Delta_{3}\right)$.

\section{Classification of primary Spin $v$ representations and some consequence}

We continue using the previous notation and complete the classification of symmetric pairs with primary Spin $v$ representations. Let $(\mathfrak{g}, \theta)$ be a reduced involutory complex semisimple Lie algebra, and $\mathfrak{g}=\mathfrak{k} \oplus \mathfrak{p}$ be the corresponding Cartan decomposition. Let $B_{\mathfrak{g}}$ be the Killing form on $\mathfrak{g}$. Let $\mathfrak{h}=\mathfrak{t} \oplus \mathfrak{a}$ be a maximally compact Cartan subalgebra of $(\mathfrak{g}, \theta)$. Let

$$
\operatorname{Spin} v: \mathfrak{k} \rightarrow \text { End } S
$$

be the representation defined as the composition of the isotropy representation $v: \mathfrak{k} \rightarrow \mathfrak{s o}(\mathfrak{p})$ with the spin representation Spin $: \mathfrak{s o}(\mathfrak{p}) \rightarrow$ End $S$, as in (2-7). Let

$$
\beta: \mathfrak{k} \rightarrow \text { End } \wedge \mathfrak{p}
$$

be the derivation extension of $v: \mathfrak{k} \rightarrow \mathfrak{s o}(\mathfrak{p})$, as in Equation (2-5).

From now on, we fix a system $P_{\mathfrak{k}}$ of positive roots of $\Delta(\mathfrak{k}, \mathfrak{t})$. Let $C\left(P_{\mathfrak{k}}\right)$ be the set of $\theta$-stable positive root systems $P$ of $\Delta(\mathfrak{g}, \mathfrak{h})$ such that $p(P) \supseteq P_{\mathfrak{k}}$. For a 
subset $Q \subseteq \Delta(\mathfrak{g}, \mathfrak{h})$ or $Q \subseteq \Delta(\mathfrak{g}, \mathfrak{t})$, we set $\rho(Q)=\frac{1}{2} \sum_{\alpha \in Q} \alpha$. For $P \in C\left(P_{\mathfrak{k}}\right)$, let $\rho_{n}(P)=\rho(P)-\rho\left(P_{\mathfrak{k}}\right)$. Because $P$ is $\theta$-stable, $\rho(P)$ and $\rho_{n}(P)$ are both in $\mathfrak{t}^{*}$. Recall that $l_{0}=\operatorname{dim} \mathfrak{a}$.

Lemma 4.1 [Wallach 1988, Lemma 9.3.2]. Let $\operatorname{Spin} v: \mathfrak{k} \rightarrow$ End $S$ be the representation defined as in (4-1). Then

$$
\operatorname{Spin} v=\sum_{P \in C\left(P_{\mathfrak{k}}\right)} 2^{\left[\left(l_{0}+1\right) / 2\right]} \pi_{\rho_{n}(P)},
$$

where $\pi_{\rho_{n}(P)}$ denotes the irreducible representation of $\mathfrak{k}$ with highest weight $\rho_{n}(P)$.

Remark 4.2. For Wallach, the definition of the Spin $v$ representation is slightly different from ours. When $\operatorname{dim} \mathfrak{p}$ is even, the two definitions agree. When $\operatorname{dim} \mathfrak{p}$ is odd, his $S$ is the spin representation $S_{1}$ of $\mathfrak{s o}(\mathfrak{p})$ in our Equation (2-10). This results in the difference of the scalar in Lemma 4.1; the scalar in Lemma 9.3.2 of [Wallach 1988] is $2^{\left[l_{0} / 2\right]}$.

In terms of the restricted root system $\Delta(\mathfrak{g}, \mathfrak{t})$, we get an equivalent statement which is convenient to use. Let

$$
D\left(P_{\mathfrak{k}}\right)=\left\{p(P) \mid P \in C\left(P_{\mathfrak{k}}\right)\right\}
$$

be the set of the positive root systems of $\Delta(\mathfrak{g}, \mathfrak{t})$ containing $P_{\mathfrak{k}}$. Remark 3.2 implies that the projection $p$ sets up a one-to-one correspondence between $C\left(P_{\mathfrak{k}}\right)$ and $D\left(P_{\mathfrak{k}}\right)$. For $P^{\prime}=p(P) \in D\left(P_{\mathfrak{k}}\right)$, define $\rho_{n}(p(P))=\rho_{n}(P)$.

Lemma 4.3. Let $\operatorname{Spin} v: \mathfrak{k} \rightarrow$ End $S$ be the representation defined as in (4-1). Then

$$
\operatorname{Spin} v=\sum_{P \in D\left(P_{\mathfrak{k}}\right)} 2^{\left[\left(l_{0}+1\right) / 2\right]} \pi_{\rho_{n}(P)} .
$$

By this lemma, Spin $v$ is primary if and only if card $D\left(P_{\mathfrak{k}}\right)=1$, that is, if there exists only one positive root system of $\Delta(\mathfrak{g}, \mathfrak{t})$ containing $P_{\mathfrak{k}}$. Note that $\Delta(\mathfrak{k}, \mathfrak{t})$ and $\Delta(\mathfrak{g}, \mathfrak{t})$ are both root systems in $\mathfrak{t}_{\mathbb{R}}^{*}$, and $\Delta(\mathfrak{k}, \mathfrak{t})$ is a root subsystem of $\Delta(\mathfrak{g}, \mathfrak{t})$. Let $W_{\mathfrak{g}}$ and $W_{\mathfrak{k}}$ be the Weyl groups of $\Delta(\mathfrak{g}, \mathfrak{t})$ and $\Delta(\mathfrak{k}, \mathfrak{t})$. Then $W_{\mathfrak{k}}$ is a subgroup of $W_{\mathfrak{g}}$. Let $\mathscr{C}$ be the Weyl chamber relative to $P_{\mathfrak{k}}$. It is clear that card $D\left(P_{\mathfrak{k}}\right)$ equals the number of Weyl chambers of $\Delta(\mathfrak{g}, \mathfrak{t})$ contained in $\mathscr{C}$, which also equals the index of $W_{\mathfrak{k}}$ in $W_{\mathfrak{g}}$.

For a nonreduced root system $\Delta$, we call a subset $\Delta_{0} \subset \Delta$ a reduced root system of $\Delta$ if $\Delta_{0}$ is a reduced root system and contains a multiple of $\alpha$ for any $\alpha \in \Delta$. (Such a multiple must be $\alpha / 2, \alpha$ or $2 \alpha$.) For example, $B_{n}$ and $C_{n}$ are reduced root systems of $(B C)_{n}$. Obviously, for a reduced root system $\Delta_{0}$ of $\Delta$, the Weyl groups of $\Delta_{0}$ and $\Delta$ are the same. Therefore, if $\Delta(\mathfrak{g}, \mathfrak{t})$ is reduced, card $D\left(P_{\mathfrak{k}}\right)=1$ if and 
only if $\Delta(\mathfrak{k}, \mathfrak{t})=\Delta(\mathfrak{g}, \mathfrak{t})$. If $\Delta(\mathfrak{g}, \mathfrak{t})$ is nonreduced, card $D\left(P_{\mathfrak{k}}\right)=1$ if and only if $\Delta(\mathfrak{k}, \mathfrak{t})$ is a reduced root system of $\Delta(\mathfrak{g}, \mathfrak{t})$.

Assume that Spin $v$ is primary. There exists only one positive root system $P$ of $\Delta(\mathfrak{g}, \mathfrak{t})$ containing $P_{\mathfrak{k}}$. We define $\rho_{n}=\rho_{n}(P)$. Then Spin $v$ is primary of type $\pi_{\rho_{n}}$. Let $P_{\mathfrak{p}}=P \cap \Delta(\mathfrak{p}, \mathfrak{t})$. Then $\Delta(\mathfrak{p}, \mathfrak{t})=P_{\mathfrak{p}} \sqcup-P_{\mathfrak{p}}$ and $\rho_{n}=\rho_{n}(P)=\rho\left(P \backslash P_{\mathfrak{k}}\right)=\rho\left(P_{\mathfrak{p}}\right)$. Summarizing:

Lemma 4.4. The representation Spin $v$ is primary if and only if $\Delta(\mathfrak{k}, \mathfrak{t})$ equals $\Delta(\mathfrak{g}, \mathfrak{t})$ or is a reduced root system of $\Delta(\mathfrak{g}, \mathfrak{t})$. In this case, Spin $v$ is primary of type $\pi_{\rho_{n}}$.

Remark 4.5. Let $r_{0}=$ card $P_{\mathfrak{p}}$. Considering Lemma 3.4, one has

$$
\operatorname{dim} \mathfrak{p}=l_{0}+2 r_{0} .
$$

Hence, when Spin $v$ is primary, by applying Equations (2-9) and (2-11) one has

$$
\bigwedge \mathfrak{p} \cong 2^{l_{0}} V_{\rho_{n}} \otimes V_{\rho_{n}},
$$

as $\mathfrak{k}$-modules. Comparing the dimensions of $\bigwedge \mathfrak{p}$ and $2^{l_{0}} V_{\rho_{n}} \otimes V_{\rho_{n}}$, we get that

$$
\operatorname{dim} V_{\rho_{n}}=2^{r_{0}} .
$$

Lemma 4.6. Let $\mathfrak{g}=\mathfrak{k} \oplus \mathfrak{p}$ be the Cartan decomposition corresponding to $\theta$. If Spin $v$ is primary, then rank $\mathfrak{g}>\operatorname{rank} \mathfrak{k}$ and the action of $\theta$ on $\Gamma$ is nontrivial, where $(\Gamma, \theta)$ is the Dynkin Diagram of $(\mathfrak{g}, \theta)$.

Proof. If rank $\mathfrak{g}=$ rank $\mathfrak{k}$, the Cartan subalgebra $\mathfrak{t}$ of $\mathfrak{k}$ is also that of $\mathfrak{g}$. So $\Delta(\mathfrak{g}, \mathfrak{t})$ and $\Delta(\mathfrak{k}, \mathfrak{t})$ are both irreducible and reduced root systems. Since $\Delta(\mathfrak{g}, \mathfrak{t}) \supsetneq \Delta(\mathfrak{k}, \mathfrak{t})$, Spin $v$ cannot be primary by Lemma 4.4. Let $(\Gamma, \theta)$ be the Dynkin diagram of $(\mathfrak{g}, \theta)$. If rank $\mathfrak{g}>\operatorname{rank} \mathfrak{k}$, then $\mathfrak{a}$ is nonzero and the action of $\theta$ on a $\theta$-stable positive root system of $\Delta(\mathfrak{g}, \mathfrak{h})$ is nontrivial, so the action of $\theta$ on $\Gamma$ is nontrivial. Conversely, if the action of $\theta$ on $\Gamma$ is nontrivial, $\operatorname{rank} \mathfrak{g}>\operatorname{rank} \mathfrak{k}$.

Next we classify the symmetric pairs $(\mathfrak{k}, \mathfrak{g})$ with primary $\operatorname{Spin} v$ representations under the condition that $\mathfrak{g}$ be simple.

Proposition 4.7. Let $(\mathfrak{g}, \theta)$ be an involutory complex simple Lie algebra and $(\mathfrak{k}, \mathfrak{g})$ be the corresponding symmetric pair. Then $\operatorname{Spin} v$ is primary if and only if $(\mathfrak{k}, \mathfrak{g})$ is one of the following:

$$
\begin{array}{ll}
(\mathfrak{s o}(2 n+1, \mathbb{C}), \mathfrak{s l}(2 n+1, \mathbb{C})), & n \geq 1 \\
(\mathfrak{s p}(n, \mathbb{C}), \mathfrak{s l}(2 n, \mathbb{C})), & n \geq 2 ; \\
(\mathfrak{s o}(2 n+1, \mathbb{C}), \mathfrak{s o}(2 n+2, \mathbb{C})), & n \geq 3 \\
\left(F_{4}, E_{6}\right) . &
\end{array}
$$


Proof. In view of Lemma 4.6, only those $\mathfrak{g}$ with a Dynkin diagram admitting a nontrivial involutory diagram automorphism may have primary Spin $v$ representations. In these cases, $\theta$ is an outer automorphism of $\mathfrak{g}$. Since $\mathfrak{g}$ is simple, $\mathfrak{g}$ must be of type $A_{n}$ with $n \geq 2, D_{n}$ with $n \geq 4$, or $E_{6}$; in each case $\theta$ is the obvious one. In each case the roots have the same length. First, we compute the Dynkin diagram of $\Delta(\mathfrak{g}, \mathfrak{t})=p(\Delta(\mathfrak{g}, \mathfrak{h}))$ by using the fact that $p$ maps the simple roots of $\Delta^{+}(\mathfrak{g}, \mathfrak{h})$ to the simple roots of $\Delta^{+}(\mathfrak{g}, \mathfrak{t})=p\left(\Delta^{+}(\mathfrak{g}, \mathfrak{h})\right)$.

For any $\alpha \in \Delta(\mathfrak{g}, \mathfrak{h})$, we use $\alpha^{\prime}$ to denote $p(\alpha) \in \Delta(\mathfrak{g}, \mathfrak{t})$. Let $\Pi$ be a $\theta$-stable simple root system in $\Delta(\mathfrak{g}, \mathfrak{h})$. Let $\alpha^{\prime}, \gamma^{\prime}$ be two different simple roots in $p(\Pi)$, and compute $\left\langle\alpha^{\prime}, \gamma^{\prime}\right\rangle\left\langle\gamma^{\prime}, \alpha^{\prime}\right\rangle$. There are three situations:

(1) If $\alpha^{\prime}=\alpha$ and $\gamma^{\prime}=\gamma$, then $\left\langle\alpha^{\prime}, \gamma^{\prime}\right\rangle\left\langle\gamma^{\prime}, \alpha^{\prime}\right\rangle=\langle\alpha, \gamma\rangle\langle\gamma, \alpha\rangle$.

(2) If $\alpha^{\prime}=\alpha$ and $\gamma^{\prime} \neq \gamma$, then there are two subcases:

(a) If $(\alpha, \gamma)=0$, then $\left(\alpha^{\prime}, \gamma^{\prime}\right)=0$ and $\left\langle\alpha^{\prime}, \gamma^{\prime}\right\rangle\left\langle\gamma^{\prime}, \alpha^{\prime}\right\rangle=0$.

(b) If $(\alpha, \gamma) \neq 0$, then $(\gamma, \theta \gamma)=0$ and

$$
\begin{aligned}
\left\langle\alpha^{\prime}, \gamma^{\prime}\right\rangle\left\langle\gamma^{\prime}, \alpha^{\prime}\right\rangle & =\frac{2\left(\alpha^{\prime}, \gamma^{\prime}\right)}{\left(\gamma^{\prime}, \gamma^{\prime}\right)} \cdot \frac{2\left(\gamma^{\prime}, \alpha^{\prime}\right)}{\left(\alpha^{\prime}, \alpha^{\prime}\right)}=\frac{4(2 \alpha, \gamma+\theta \gamma)^{2}}{(\gamma+\theta \gamma, \gamma+\theta \gamma)(2 \alpha, 2 \alpha)} \\
& =2\langle\alpha, \gamma\rangle\langle\gamma, \alpha\rangle .
\end{aligned}
$$

(3) If $\alpha^{\prime} \neq \alpha$ and $\gamma^{\prime} \neq \gamma$, we may assume that $(\alpha, \theta \gamma)=0$, and then

$$
\begin{aligned}
\left\langle\alpha^{\prime}, \gamma^{\prime}\right\rangle\left\langle\gamma^{\prime}, \alpha^{\prime}\right\rangle & =\frac{2\left(\alpha^{\prime}, \gamma^{\prime}\right)}{\left(\gamma^{\prime}, \gamma^{\prime}\right)} \cdot \frac{2\left(\gamma^{\prime}, \alpha^{\prime}\right)}{\left(\alpha^{\prime}, \alpha^{\prime}\right)} \\
& =\frac{4(\alpha+\theta \alpha, \gamma+\theta \gamma)^{2}}{(\gamma+\theta \gamma, \gamma+\theta \gamma)(\alpha+\theta \alpha, \alpha+\theta \alpha)} .
\end{aligned}
$$

There are again two subcases:

(a) If $(\alpha, \theta \alpha)=0$ and $(\gamma, \theta \gamma)=0$, then

$$
\left\langle\alpha^{\prime}, \gamma^{\prime}\right\rangle\left\langle\gamma^{\prime}, \alpha^{\prime}\right\rangle=\frac{4(\alpha, \gamma)^{2}}{(\gamma, \gamma)(\alpha, \alpha)}=\langle\alpha, \gamma\rangle\langle\gamma, \alpha\rangle
$$

(b) If one of $(\alpha, \theta \alpha)$ and $(\gamma, \theta \gamma)$ is 0 , then, assuming $(\alpha, \theta \alpha)=0$, we have $(\gamma, \theta \gamma)=-\frac{1}{2}(\gamma, \gamma)$ and

$$
\left\langle\alpha^{\prime}, \gamma^{\prime}\right\rangle\left\langle\gamma^{\prime}, \alpha^{\prime}\right\rangle=\frac{8(\alpha, \gamma)^{2}}{(\gamma, \gamma)(\alpha, \alpha)}=2\langle\alpha, \gamma\rangle\langle\gamma, \alpha\rangle
$$

Thus, when the Dynkin diagram of $\Pi$ is $A_{2 n}, A_{2 n-1}, D_{n+1}$ or $E_{6}$, the Dynkin diagram of $p(\Pi)$ is respectively $B_{n}, C_{n}, B_{n}$ or $F_{4}$, as shown in Figure 1.

But when $\Delta(\mathfrak{g}, \mathfrak{h})$ is $A_{2 n}$, the root system $p(\Delta(\mathfrak{g}, \mathfrak{h}))$ is not $B_{n}$, but $(B C)_{n}$. Indeed, if $\alpha$ is the simple root from (a) of Figure 1 , then $\alpha+p(\alpha)=2 \alpha^{\prime}$ is in 

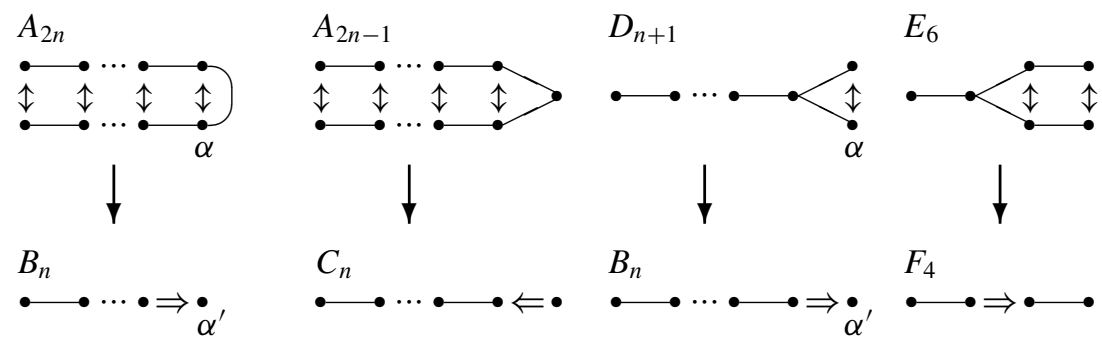

\section{Figure 1}

$p(\Delta(\mathfrak{g}, \mathfrak{h}))$. When $\Delta(\mathfrak{g}, \mathfrak{h})$ is $D_{n+1}$, the root system $p(\Delta(\mathfrak{g}, \mathfrak{h}))$ is just $B_{n}$. Indeed, for $\alpha$ from (c) of Figure 1, $2 \alpha^{\prime}$ is not in $p(\Delta(\mathfrak{g}, \mathfrak{h}))$. Then we get

$$
\begin{array}{ll}
p\left(A_{2 n}\right)=(B C)_{n}, \quad n \geq 1 ; & p\left(A_{2 n-1}\right)=C_{n}, \quad n \geq 2 ; \\
p\left(D_{n+1}\right)=B_{n}, \quad n \geq 3 ; & p\left(E_{6}\right)=F_{4} .
\end{array}
$$

One knows that such $(\mathfrak{g}, \theta)$ and noncompact noncomplex real simple Lie algebras correspond to each other and all such Lie algebras have been classified by their Vogan diagrams.

For $\left(A_{2 n}, \theta\right)$, only $\mathfrak{s l}(2 n+1, \mathbb{R})$ 's Dynkin diagram is $\left(A_{2 n}, \theta\right)$. Now $\mathfrak{k}_{0}=\mathfrak{s o}(2 n+$ 1) and $\Delta(\mathfrak{k}, \mathfrak{t})=B_{n}$ is a reduced root system of $\Delta(\mathfrak{g}, \mathfrak{t})=(B C)_{n}$, so Spin $v$ is primary. In this case,

$$
(\mathfrak{k}, \mathfrak{g})=(\mathfrak{s o}(2 n+1, \mathbb{C}), \mathfrak{s l}(2 n+1, \mathbb{C})), \quad n \geq 1 .
$$

The Dynkin diagrams of $\mathfrak{s l}(2 n, \mathbb{R})$ and $\mathfrak{s u}^{*}(2 n)$ are $\left(A_{2 n-1}, \theta\right)$. If $\mathfrak{g}_{0}=\mathfrak{s l}(2 n, \mathbb{R})$, then $\mathfrak{k}_{0}=\mathfrak{s o}(2 n)$, and $\Delta(\mathfrak{k}, \mathfrak{t})=D_{n}$ does not equal $\Delta(\mathfrak{g}, \mathfrak{t})=C_{n}$, so the Spin $v$ representation is not primary. If $\mathfrak{g}_{0}=\mathfrak{s u}^{*}(2 n)$, then $\mathfrak{k}_{0}=\mathfrak{s p}(n)$ and $\Delta(\mathfrak{k}, \mathfrak{t})=C_{n}=$ $\Delta(\mathfrak{g}, \mathfrak{t})$, so the $\operatorname{Spin} v$ representation is primary. In this case,

$$
(\mathfrak{k}, \mathfrak{g})=(\mathfrak{s p}(n, \mathbb{C}), \mathfrak{s l}(2 n, \mathbb{C})), \quad n \geq 2 .
$$

The Dynkin diagrams of $\mathfrak{s o}(2 r+1,2 n-2 r+1)$, with $n \geq 3$ and $0 \leq r \leq[n / 2]$, are $\left(D_{n+1}, \theta\right)$. If $\mathfrak{g}_{0}=\mathfrak{s o}(2 r+1,2 n-2 r+1)$, then $\mathfrak{k}_{0}=\mathfrak{s o}(2 r+1) \oplus \mathfrak{s o}(2 n-2 r+1)$ and $\Delta(\mathfrak{k}, \mathfrak{t})=B_{r} \sqcup B_{n-r}$. Therefore $\Delta(\mathfrak{k}, \mathfrak{t})=\Delta(\mathfrak{g}, \mathfrak{t})=B_{n}$ if and only if $r=0$. So, when $\mathfrak{g}_{0}=\mathfrak{s o}(1,2 n+1)$, or $(\mathfrak{k}, \mathfrak{g})=(\mathfrak{s o}(2 n+1, \mathbb{C}), \mathfrak{s o}(2 n+2, \mathbb{C}))$ with $n \geq 3$, the Spin $v$ representation is primary.

The Dynkin diagrams of $\mathfrak{e}_{6}(-26)$ and $\mathfrak{e}_{6}(6)$ are $\left(E_{6}, \theta\right)$. If $\mathfrak{g}_{0}=\mathfrak{e}_{6}(-26)$ then $\mathfrak{k}_{0}=\mathfrak{f}_{4}$. Then $\Delta(\mathfrak{k}, \mathfrak{t})=F_{4}=\Delta(\mathfrak{g}, \mathfrak{t})$ and the Spin $v$ representation is primary. In this case $(\mathfrak{k}, \mathfrak{g})=\left(F_{4}, E_{6}\right)$, where we use $E_{6}$ (respectively, $\left.F_{4}\right)$ to denote the simple complex Lie algebra of type $E_{6}$ (respectively, $F_{4}$ ). If $\mathfrak{g}_{0}=\mathfrak{e}_{6}(6)$, then $\mathfrak{k}_{0}=\mathfrak{s p}(4)$. Obviously, $\Delta(\mathfrak{k}, \mathfrak{t})=C_{4}$ does not equal $\Delta(\mathfrak{g}, \mathfrak{t})$, so the Spin $v$ representation is not primary. 
Remark 4.8. The isotropy representations $v$ of $\left(F_{4}, E_{6}\right)$, of $(\mathfrak{s p}(n, \mathbb{C}), \mathfrak{s l}(2 n, \mathbb{C}))$, and of $(\mathfrak{s o}(2 n+1, \mathbb{C}), \mathfrak{s o}(2 n+2, \mathbb{C}))$, and are the little adjoint representations (that is, the representation having the short dominant root of $\mathfrak{k}$ as the highest weight).

Now we will deal with the general case when $\mathfrak{g}$, from a symmetric pair $(\mathfrak{k}, \mathfrak{g})$, is semisimple.

Suppose $\mathfrak{g}=\mathfrak{g}_{1} \oplus \cdots \oplus \mathfrak{g}_{n}$ is a decomposition of $\mathfrak{g}$ into direct sum of ideals and $\theta\left(\mathfrak{g}_{i}\right)=\mathfrak{g}_{i}$ for all $i$. Let $\mathfrak{g}=\mathfrak{k} \oplus \mathfrak{p}$, and $\mathfrak{g}_{i}=\mathfrak{k}_{i} \oplus \mathfrak{p}_{i}$ be the Cartan decomposition. Then $\mathfrak{k}=\mathfrak{k}_{1} \oplus \cdots \oplus \mathfrak{k}_{n}$ and $\mathfrak{p}=\mathfrak{p}_{1} \oplus \cdots \oplus \mathfrak{p}_{n}$.

Lemma 4.9. The representation $\operatorname{Spin} v: \mathfrak{k} \rightarrow$ End $S$ is primary if and only if $\operatorname{Spin} v_{i}$ : $\mathfrak{k}_{i} \rightarrow$ End $S_{i}$ is primary for all $i$.

Proof. Let $\mathfrak{t}_{i}$ be a Cartan subalgebra of $\mathfrak{k}_{i}$. Then $\mathfrak{t}=\bigoplus_{i} \mathfrak{t}_{i}$ is a Cartan subalgebra of $\mathfrak{k}$. We know that $\Delta(\mathfrak{g}, \mathfrak{t})=\bigsqcup_{i} \Delta\left(\mathfrak{g}_{i}, \mathfrak{t}_{i}\right)$ and $\Delta(\mathfrak{k}, \mathfrak{t})=\bigsqcup_{i} \Delta\left(\mathfrak{k}_{i}, \mathfrak{t}_{i}\right)$, as well as $\Delta\left(\mathfrak{g}_{i}, \mathfrak{t}_{i}\right) \perp \Delta\left(\mathfrak{g}_{j}, \mathfrak{t}_{j}\right)$ and $\Delta\left(\mathfrak{k}_{i}, \mathfrak{t}_{i}\right) \perp \Delta\left(\mathfrak{k}_{j}, \mathfrak{t}_{j}\right)$ for $i \neq j$. Fix a positive root system $P_{\mathfrak{k}}$ of $\Delta(\mathfrak{k}, \mathfrak{t})$; then $P_{\mathfrak{k}_{i}}=P \cap \Delta\left(\mathfrak{k}_{i}, \mathfrak{t}_{i}\right)$ is a positive root system of $\Delta\left(\mathfrak{k}_{i}, \mathfrak{t}_{i}\right)$. If $P_{i}$ is a positive root system of $\Delta\left(\mathfrak{g}_{i}, \mathfrak{t}_{i}\right)$, then $P=\bigsqcup_{i} P_{i}$ is a positive root system of $\Delta(\mathfrak{g}, \mathfrak{t})$, and all the positive root systems of $\Delta(\mathfrak{g}, \mathfrak{t})$ can be obtained in this way. Then it is clear that card $D\left(P_{\mathfrak{k}}\right)=\prod_{i}$ card $D\left(P_{\mathfrak{k}_{i}}\right)$. Since card $D\left(P_{\mathfrak{k}}\right)=1$ if and only if card $D\left(P_{\mathfrak{k}_{i}}\right)=1$ for all $\mathrm{i}$, it follows that Spin $v$ is primary if and only if Spin $v_{i}$ are primary for all $i$.

Thus, we need only find all the "minimal" reduced symmetric pairs such that the corresponding Spin $v$ representations are primary. The complex semisimple Lie algebra $\mathfrak{g}$ can be uniquely decomposed into a direct sum of simple ideals. Since $\theta$ is an involutory automorphism, it acts on the set of simple ideals and it must fix some of them and permute in 2-cycles the rest of them. So one has

Lemma 4.10. The semisimple Lie algebra $\mathfrak{g}$ can be decomposed as

$$
\mathfrak{g}=\left(\mathfrak{g}_{1} \oplus \mathfrak{g}_{1}\right) \oplus\left(\mathfrak{g}_{2} \oplus \mathfrak{g}_{2}\right) \oplus \cdots \oplus\left(\mathfrak{g}_{s} \oplus \mathfrak{g}_{s}\right) \oplus \mathfrak{g}_{s+1} \oplus \cdots \oplus \mathfrak{g}_{s+l},
$$

where every $\mathfrak{g}_{i}$ is a simple ideal of $\mathfrak{g}$ such that: when $1 \leq i \leq s$, we have $\theta\left(\mathfrak{g}_{i} \oplus \mathfrak{g}_{i}\right)=$ $\mathfrak{g}_{i} \oplus \mathfrak{g}_{i}$ and $\theta$ interchanges the two $\mathfrak{g}_{i}$; and, when $s<i \leq s+j$, we have $\theta\left(\mathfrak{g}_{i}\right)=\mathfrak{g}_{i}$.

Note that $\theta$ must be an isomorphism of the two $\mathfrak{g}_{i}$ 's when $1 \leq i \leq s$, but that $\theta$ cannot be the identity on $\mathfrak{g}_{i}$ when $s<i \leq s+j$, because $(\mathfrak{g}, \theta)$ is reduced.

Lemma 4.11. If $\mathfrak{g}=\mathfrak{g}_{1} \oplus \mathfrak{g}_{1}$, with $\mathfrak{g}_{1}$ a simple ideal, and $\theta$ interchanges the two ideals, then Spin $v$ is primary of type $\pi_{\rho_{\mathfrak{g}_{1}}}$.

Proof. Because $\theta$ interchanges the two ideals, $\theta(X, Y)=\left(\varphi^{-1}(Y), \varphi(X)\right)$ for some isomorphism $\varphi$ of $\mathfrak{g}_{1}$. Recall the definition of an isomorphism of involutory Lie algebras from page 39. Define an isomorphism $\psi=(\varphi, I): \mathfrak{g}_{1} \oplus \mathfrak{g}_{1} \rightarrow \mathfrak{g}_{1} \oplus \mathfrak{g}_{1}$ by $(X, Y) \mapsto(\varphi(X), Y)$. Then $(\mathfrak{g}, \theta)$ is isomorphic to $\left(\mathfrak{g}, \theta^{\prime}\right)$ through $\psi$, where 
$\theta^{\prime}(X, Y)=(Y, X)$. With respect to $\theta^{\prime}$, we have $\mathfrak{k}=\left\{(X, X) \mid X \in \mathfrak{g}_{1}\right\} \cong \mathfrak{g}_{1}$ and $\mathfrak{p}=\left\{(X,-X) \mid X \in \mathfrak{g}_{1}\right\}$, and the representation $v$ is just the adjoint representation of $\mathfrak{g}_{1}$. By Theorem 40 of [Kostant 1997], Spin $v$ is primary of type $\pi_{\rho_{\mathfrak{g}_{1}}}$.

Remark 4.12. In this case it can be computed directly that $\rho_{n}=\rho_{\mathfrak{g}_{1}}$.

Combining the results from Proposition 4.7 to Lemma 4.11, we get:

Theorem 4.13. Let $(\mathfrak{g}, \theta)$ be a reduced involutory complex semisimple Lie algebra and $(\mathfrak{k}, \mathfrak{g})$ be the corresponding symmetric pair. Then Spin $v$ is primary if and only if $(\mathfrak{k}, \mathfrak{g})$ is one of the following:

(1) $(\mathfrak{s o}(2 n+1, \mathbb{C}), \mathfrak{s l}(2 n+1, \mathbb{C})), \quad n \geq 1 ;$ $(\mathfrak{s p}(n, \mathbb{C}), \mathfrak{s l}(2 n, \mathbb{C})), n \geq 2$ $(\mathfrak{s o}(2 n+1, \mathbb{C}), \mathfrak{s o}(2 n+2, \mathbb{C})), \quad n \geq 3$; $\left(F_{4}, E_{6}\right)$;

(2) $\mathfrak{g}=\mathfrak{g}_{1} \oplus \mathfrak{g}_{1}$ and $\mathfrak{k}=\left\{(X, X) \mid X \in \mathfrak{g}_{1}\right\}$, where $\mathfrak{g}_{1}$ is a complex simple Lie algebra;

(3) direct sums of (k, $\mathfrak{g})$ in (1) and (2).

When $(\mathfrak{k}, \mathfrak{g})$ is as above, Spin $v$ is primary of type $\pi_{\rho_{n}}$.

Combining Proposition 2.4 and Theorem 4.13, we get our main result:

Theorem 4.14. Assume that $v: \mathfrak{r} \rightarrow \mathfrak{s o}(\mathfrak{p})$ is the complexified differential of a faithful $B_{\mathfrak{p}}$-orthogonal representation of a compact Lie group and that $\mathfrak{p}^{\mathfrak{r}}=0$. Assume that Spin $v$ is primary. Then $\mathfrak{g}=\mathfrak{r} \oplus \mathfrak{p}$ has a semisimple Lie algebra structure such that $\mathfrak{r}$ is a Lie subalgebra of $\mathfrak{g}$, that $(\mathfrak{r}, \mathfrak{g})$ is a reduced symmetric pair, and that $v$ is the isotropy representation of $(\mathfrak{r}, \mathfrak{g})$. Furthermore, the symmetric pair $(\mathfrak{r}, \mathfrak{g})$ must be one of those listed in Theorem 4.13, and $\operatorname{Spin} v$ is primary of type $\pi_{\rho_{n}}$.

Recalling Remark 4.5, we get, as a corollary of Proposition 20 of [Kostant 1997]:

Corollary 4.15. Let $(\mathfrak{k}, \mathfrak{g})$ be a symmetric pair in the list of Theorem 4.13. Then the corresponding Spin $v$ representation is primary of type $\pi_{\rho_{n}}$. Let $\mathfrak{k}$ act on $\wedge \mathfrak{p}$ via $\beta$, and let $J=(\bigwedge \mathfrak{p})^{\mathfrak{k}}$. Then $\operatorname{dim} V_{\rho_{n}}=2^{r_{0}}$ and $\operatorname{dim} J=2^{l_{0}}$. As a subalgebra of $C(\mathfrak{p}), J$ is isomorphic to a matrix algebra if $l_{0}$ is even, and is isomorphic to a sum of two matrix algebras if $l_{0}$ is odd. Furthermore,

$$
C(\mathfrak{p}) \cong \text { End } V_{\rho_{n}} \otimes J
$$

as algebras. Finally, as $\mathfrak{k}$-modules,

$$
\bigwedge \mathfrak{p} \cong 2^{l_{0}} V_{\rho_{n}} \otimes V_{\rho_{n}}
$$




\section{Acknowledgements}

The main result of this paper is part of my Ph.D. thesis at the Hong Kong University of Science and Technology. I heartily thank my supervisor Jing-Song Huang for drawing my attention to [Kostant 1997] and for his great generosity in sharing his immense knowledge with me. I am also very grateful to Bin-Yong Sun for conversations about the restricted root system. Finally, I thank Jiang-Hua Lu for giving me some good suggestions when she read the paper.

\section{References}

[Kostant 1961] B. Kostant, "Lie algebra cohomology and the generalized Borel-Weil theorem", Ann. of Math. (2) 74 (1961), 329-387. MR 26 \#265 Zbl 0134.03501

[Kostant 1965] B. Kostant, "Eigenvalues of the Laplacian and commutative Lie subalgebras", Topology 3:suppl. 2 (1965), 147-159. MR 29 \#4839 Zbl 0134.03504

[Kostant 1997] B. Kostant, "Clifford algebra analogue of the Hopf-Koszul-Samelson theorem, the $\rho$-decomposition $C(\mathfrak{g})=$ End $V_{\rho} \otimes C(P)$, and the $\mathfrak{g}$-module structure of $\bigwedge \mathfrak{g}$ ", Adv. Math. 125:2 (1997), 275-350. MR 98k:17009 Zbl 0882.17002

[Kostant 1999] B. Kostant, "A cubic Dirac operator and the emergence of Euler number multiplets of representations for equal rank subgroups", Duke Math. J. 100:3 (1999), 447-501. MR 2001k:22032 Zbl 0952.17005

[Kostant 2000] B. Kostant, "On $\bigwedge \mathfrak{g}$ for a semisimple Lie algebra $\mathfrak{g}$, as an equivariant module over the symmetric algebra $S(\mathfrak{g})$ ", pp. 129-144 in Analysis on homogeneous spaces and representation theory of Lie groups (Okayama-Kyoto, 1997), edited by T. Kobayashi et al., Adv. Stud. Pure Math. 26, Math. Soc. Japan, Tokyo, 2000. MR 2001g:17009 Zbl 0986.17002

[Panyushev 2001] D. I. Panyushev, "The exterior algebra and 'spin' of an orthogonal g-module", Transform. Groups 6:4 (2001), 371-396. MR 2002k:17017 Zbl 0994.17004

[Wallach 1988] N. R. Wallach, Real reductive groups, vol. I, Pure and Applied Mathematics 132, Academic Press, Boston, 1988. MR 89i:22029 Zbl 0666.22002

Received October 15, 2004. Revised August 23, 2005.

GANG HAN

MAILBOX 1511

Center of Mathematical Sciences

ZHEJIANG UNIVERSITY

HANGZHOU 310027

CHINA

mathhg@cms.zju.edu.cn

The diagram on page 38 was produced with Paul Taylor's diagrams.sty package. 